\title{
LVG Hamster
}

National Cancer Institute

\section{Source}

National Cancer Institute. LVG Hamster. NCI Thesaurus. Code C77094.

Derived from the orig inal group of Syrian hamsters captured by Dr. Israel Aharoni in 1930 and imported into the United States in 1938, followed by colony derivation at Lakeview in 1949 and 1951 and to Charles River in 1969, where the strain is propagated today. This breed is used in toxicological studies and is prone to amyloidosis of the kidneys, liver, and adrenal glands after twelve months of age. 